MATHEMATICS OF COMPUTATION

Volume 69, Number 230, Pages 815-825

S 0025-5718(99)01121-7

Article electronically published on May 21, 1999

\title{
EXPLICIT RESOLUTIONS OF CUBIC CUSP SINGULARITIES
}

\author{
H. G. GRUNDMAN
}

\begin{abstract}
Resolutions of cusp singularities are crucial to many techniques in computational number theory, and therefore finding explicit resolutions of these singularities has been the focus of a great deal of research. This paper presents an implementation of a sequence of algorithms leading to explicit resolutions of cusp singularities arising from totally real cubic number fields. As an example, the implementation is used to compute values of partial zeta functions associated to these cusps.
\end{abstract}

\section{INTRODUCTION}

The purpose of this paper is to present an implementation of a sequence of algorithms leading to explicit resolutions of cusp singularities arising from totally real cubic number fields. Resolutions of these singularities are crucial to many techniques in computational number theory. As an example, we use this implementation to compute values of partial zeta functions.

Although the theories are well understood, in practice determining a specific resolution of a cusp singularity is tedious at best. Let $K$ be a totally real cubic number field with ring of integers $\mathcal{O}_{K}$. We are interested in cusps of type $(M, V)$ where $M \subseteq K$ is an $\mathcal{O}_{K}$-module of rank 3 (that we will assume contains 1 ) and $V$ is a subgroup of $U_{K}^{+}$of rank 2. The computer program described here provides resolutions of these cusps, given only basic information about $M$ and $V$. A family of cusps of particular interest consists of cusps of type $\left(\mathcal{O}_{K}, U_{K}^{+}\right)$, where $U_{K}^{+}$is the group of totally positive units in $\mathcal{O}_{K}$. Another consists of cusps of type $\left(\mathcal{O}_{K}, U_{K}^{2}\right)$, the cusps of the Hilbert modular threefolds. For clarity, we describe the algorithms for $(M, V)=\left(\mathcal{O}_{K}, U_{K}^{+}\right)$, although, as explained below, the same computer program will resolve cusps of other types as well.

Finding resolutions of cusps has been the focus of a great deal of research. (See for example 1, 3, $5,7,8,11$.) The resolutions derived here are based on the work of Ehlers [5] and Thomas and Vasquez [14] and are determined in two steps. Consider the action of $U_{K}^{+}$on $\mathbf{R}_{+}^{3}$ defined by coordinate-wise multiplication via the three embeddings of $K$ into $\mathbf{R}$. The first step of the process is to find a fundamental domain for this action specified as the disjoint union of a finite number of open simplicial cones, each of which has vertices in $\mathcal{O}_{K}$. The next step is to find a specific decomposition of these cones into new cones such that the vertices of each

Received by the editor June 6, 1997 and, in revised form, June 22, 1998.

1991 Mathematics Subject Classification. Primary: 32S45, 11J30; Secondary: 11-04.

This material is based partially on work supported by the National Science Foundation under Grant No. DMS-9115349 and by the Faculty Research Fund of Bryn Mawr College. 
new cone either form a basis or can be extended to form a basis of $\mathcal{O}_{K}$. This decomposition yields a resolution of the cusp of type $\left(\mathcal{O}_{K}, U_{K}^{+}\right)$.

The generalization to cusps of the form $(M, V)$ is straightforward. For $M \neq \mathcal{O}_{K}$, a basis for $M$ needs to be given in place of a basis for $\mathcal{O}_{K}$. For $V \neq U_{K}^{+}$, the output for $\left(M, U_{K}^{+}\right)$can still be used, given generators for $V$ in terms of generators for $U_{K}^{+}$. The fundamental domain for the action of $V$ on $\mathbf{R}_{+}^{3}$ is made up of $\left[U_{K}^{+}: V\right]$ copies of the fundamental domain for the action of $U_{K}^{+}$. Thus, a decomposition of a fundamental domain for the action of $U_{K}^{+}$gives us a decomposition of a fundamental domain for the action of $V$, and therefore a resolution of the cusp $(M, V)$.

Shintani's method for computing special values of partial zeta functions begins identically with this method for resolving the cusp $\left(\mathcal{O}_{K}, U_{K}^{+}\right)$. The first step is the determination of a fundamental domain for the action of $U_{K}^{+}$acting on $\mathbf{R}_{+}^{3}$ given as a disjoint union of a finite number of open simplicial cones each with vertices in $\mathcal{O}_{K}$. Although Shintani's formula can be applied to this collection of cones, the computation is greatly simplified by first decomposing the cones as described above.

In Sections 2 and 3 we discuss the determination of a suitable fundamental domain for the action of $U_{K}^{+}$on $\mathbf{R}_{+}^{3}$ and the method for finding a suitable decomposition of the domain. In Section 4 we describe the details of the computer implementation. We describe the numeric and graphical output and present samples of each in Section [5. Finally, in Section [6 we explain Shintani's method and give the application of this program to the problem of computing values of partial zeta functions.

\section{Fundamental domains}

The first problem to address is that of finding a fundamental domain for the action of a group of totally positive units in a three-dimensional lattice (in this case, $U_{K}^{+}$in $\mathcal{O}_{K}$ ) acting on $\mathbf{R}_{+}^{3}$. The methods used here are based on work of Thomas and Vasquez 14, the relevant parts of which we summarize below.

Given $\alpha, \beta \in U_{K}^{+}$, Thomas and Vasquez call $(\alpha, \beta)$ a proper pair if $\alpha$ and $\beta$ are independent, $\{1, \alpha, \beta\}$ forms a basis for $K$ over $\mathbf{Q}$, and given $x, y, z \in \mathbf{Q}$ such that $\alpha \beta=x \alpha+y \beta+z \cdot 1, z$ is negative.

Lemma 1 (Thomas, Vasquez). For $v_{1}, \ldots, v_{k}$ independent vectors in $\mathbf{R}^{3}$, let $C\left(v_{1}, \ldots, v_{k}\right)$ be the open simplicial cone defined by

$$
C\left(v_{1}, \ldots, v_{k}\right)=\left\{t_{1} v_{1}+\cdots+t_{k} v_{k} \mid t_{i}>0\right\} .
$$

Given a proper pair of units $(\alpha, \beta)$ that generate $U_{K}^{+}$, a fundamental domain for the action of $U_{K}^{+}$on $\mathbf{R}_{+}^{3}$ is given by the disjoint union of

$$
C(\alpha), C(\alpha, 1), C(\alpha, \beta), C(\alpha, \alpha \beta), C(\alpha, \alpha \beta, \beta), C(\alpha, 1, \beta) .
$$

This lemma reduces the problem of determining a fundamental domain to that of finding a proper pair of fundamental units for $U_{K}^{+}$. Again, there are a number of methods available for finding fundamental units. See, for example, 4, 10. As noted in [14], however, a method based on the work of Berwick [2] is guaranteed to produce a proper pair of units. For each $a \in K$ and for $i=1,2,3$, let $a^{(i)}$ denote the image of $a$ under the $i$-th embedding of $K$ into $\mathbf{R}$.

Theorem 2 (Berwick). Let $M$ be an arbitrary cubic order and for $i=0,1,2$, let $\mathcal{C}_{i}$ be the corresponding cylinder:

$$
\mathcal{C}_{i}=\left\{\eta \in M: \eta^{(i)}>1 \text { and }\left|\eta^{(j)}\right|<1 \text { for each } j \in\{0,1,2\}, j \neq i\right\} .
$$


(1) There exist units of $M$ in each $\mathcal{C}_{i}$.

(2) Let $\varepsilon_{i} \in \mathcal{C}_{i}$ be a unit such that $\varepsilon_{i}^{(i)} \leq \eta^{(i)}$ for each unit $\eta \in \mathcal{C}_{i}$. Then any two of the three units $\varepsilon_{0}, \varepsilon_{1}, \varepsilon_{2}$ form a fundamental system of units for $M$.

Let $K=\mathbf{Q}(\lambda)$. Then each element of $\mathcal{O}_{K}$ can be written in the form $a \lambda^{2}+b \lambda+c$ where $a, b, c \in \mathbf{Q}$. Theorem 2 gives three pairs of inequalities for each cylinder:

$$
\begin{aligned}
1<a\left(\lambda^{(i)}\right)^{2}+b \lambda^{(i)}+c & <B, \\
-1<a\left(\lambda^{(j)}\right)^{2}+b \lambda^{(j)}+c & <1, \\
-1<a\left(\lambda^{(k)}\right)^{2}+b \lambda^{(k)}+c & <1,
\end{aligned}
$$

where $B$ is some bound larger than 1 . For each cylinder, these inequalities can be "solved" to yield three pairs of inequalities for $a$ in terms of the $\lambda^{(i)}, \lambda^{(j)}$, and $\lambda^{(k)}$, or three pairs of inequalities for $b$ in terms of $a$ and the embeddings of $\lambda$. Thomas [13] used these inequalities with the assumption that $a, b, c \in \mathbf{Z}$. Here, we do not make that assumption, but use the fact that we can bound the size of the denominators of $a, b$ and $c$. With this, we can, for a given value of $B$, find all possible values of $a$. Then, for each of these, we can find all possible values of $b$ and finally, of $c$. The set of elements thus found is contained in the given cylinder. If the set contains any units of $\mathcal{O}_{K}$, something that is easily checked, the minimal unit must be $\varepsilon_{i}$.

As noted in [14], Berwick's theorem can be extended to apply to any subgroup of $U_{K}$ of maximal rank, using the same proof. For $i=1,2,3$, we let $\bar{\varepsilon}_{i}$ be the smallest (in the sense of Theorem 21) totally positive unit in the cylinder $\mathcal{C}_{i}$. Proposition 1 in [14 implies that $(\alpha, \beta)$ is a proper pair of units, where $\alpha=\bar{\varepsilon}_{0}$ and $\beta=\bar{\varepsilon}_{1}$ if $\left(\bar{\varepsilon}_{0}\right)^{(1)}>\left(\bar{\varepsilon}_{0}\right)^{(2)}$, and $\beta=\bar{\varepsilon}_{2}$ otherwise.

\section{Decompositions}

The next problem is that of finding a suitable decomposition of the fundamental domain.

Given an open simplicial cone $C\left(v_{1}, v_{2}, v_{3}\right)$ with $v_{i}=\left(\alpha_{i}^{(0)}, \alpha_{i}^{(1)}, \alpha_{i}^{(2)}\right)$ for some $\alpha_{i} \in \mathcal{O}_{K}, i=1,2,3$, we subdivide the cone by first specifying a new element $\beta \in \mathcal{O}_{K}$ such that $w=\left(\beta^{(0)}, \beta^{(1)}, \beta^{(2)}\right)$ is in the set

$$
\left\{t_{1} v_{1}+t_{2} v_{2}+t_{3} v_{3} \mid 0 \leq t_{i}<1\right\}
$$

and such that the sum $t_{1}+t_{2}+t_{3}>0$ is minimal. There are two cases. If every $t_{i}$ is nonzero, then the point $w$ is inside the cone and we decompose $C\left(v_{1}, v_{2}, v_{3}\right)$ into the union of the cones

$$
C\left(w, v_{2}, v_{3}\right), C\left(v_{1}, w, v_{3}\right), C\left(v_{1}, v_{2}, w\right), C\left(v_{1}, w\right), C\left(v_{2}, w\right), C\left(v_{3}, w\right), C(w) .
$$

If one of the $t_{i}$, say $t_{1}$, is zero, then the point $w$ is in the cone $C\left(v_{2}, v_{3}\right)$ and we decompose $C\left(v_{1}, v_{2}, v_{3}\right)$ into the union of the cones

$$
C\left(v_{1}, w, v_{3}\right), C\left(v_{1}, v_{2}, w\right), C\left(v_{1}, w\right),
$$

and $C\left(v_{2}, v_{3}\right)$ into the union of the cones

$$
C\left(w, v_{3}\right), C\left(v_{2}, w\right), C(w) .
$$


In this latter case, the second three-dimensional cone adjacent to the cone $C\left(v_{2}, v_{3}\right)$ is also subdivided. Specifically, if the original cone is $C\left(v_{4}, v_{2}, v_{3}\right)$, it is decomposed into the union of the cones

$$
C\left(v_{4}, w, v_{3}\right), C\left(v_{4}, v_{2}, w\right), C\left(v_{4}, w\right) .
$$

Note that when $v_{2}$ and $v_{3}$ are on the boundary of the original fundamental domain, the second three-dimensional cone is actually adjacent to a translate of $C\left(v_{2}, v_{3}\right)$ and is subdivided by a translate of the point $w$, as is the translate of $C\left(v_{2}, v_{3}\right)$.

This process is then repeated recursively with each three-dimensional cone thus created. For each of the new cones, the volume of the set

$$
\left\{t_{1} v_{1}+t_{2} v_{2}+t_{3} v_{3} \mid 0 \leq t_{i}<1\right\}
$$

is a rational integer strictly less than the volume of the set corresponding to the cone that was just subdivided. As long as this volume is strictly greater than one, a new subdivision point can be found. Hence, the process terminates only when each resulting cone has volume equal to one. It is easy to see that this corresponds to the vertices of the cone forming an integral basis for $\mathcal{O}_{K}$. Thus, following [14, this process produces a resolution of the cusp $\left(\mathcal{O}_{K}, U_{K}^{+}\right)$in the sense of Ehlers [5].

Notice that the two-dimensional cones (i.e., $C(\alpha, 1), C(\alpha, \beta), C(\alpha, \alpha \beta)$, along with any generated in the decomposition process) are all decomposed in this process and therefore do not need to be dealt with separately. As explained in Section 1 . this method is very general and can be applied to cusps of other types.

The decomposition method we use was originally inspired by those used by Haspel and Vasquez in [7], but other than the inherently recursive nature of the algorithms there remains little similarity between the two methods. Specifically, our fundamental domains are triangulated by construction, thus eliminating the need for the first phase of their decomposition. Further, unlike [7], we do not choose elements of minimal trace.

\section{The COMputer Program}

The computer program requires minimal input data that can be easily computed or obtained from readily available tables. Specifically, the program requires the coefficients of a totally real cubic polynomial a root of which generates $K$ over $\mathrm{Q}$, and a basis for $\mathcal{O}_{K}$ given as integral linear combinations of powers of the root over a common denominator, $d$. We have given a number of options for output, including both textual and pictorial, some creating data designed to be computer readable for further computations. For details on these choices, with examples, see the following sections of this paper.

To find a fundamental domain for the action of $U_{K}^{+}$on $\mathbf{R}_{+}^{3}$, we first use Theorem2 as described in Section 2 to find the units $\bar{\varepsilon}_{0}, \bar{\varepsilon}_{1}, \bar{\varepsilon}_{2}$. The process is the same for each $\bar{\varepsilon}_{i}$, so let $i$ be fixed for now. Since $\mathcal{O}_{K}$ is contained in $\frac{1}{d} \mathbf{Z}[\lambda]$, the rational coefficients $a, b$, and $c$ in (11) are in $\frac{1}{d} \mathbf{Z}$. So for a fixed bound $B$, there are only a finite number of possible values for the coefficients. Starting with an initial value of $B=32$, then increasing it if necessary, the program computes all possible triples $(a, b, c)$ satisfying (11). Numeric approximations of the three real roots are obtained using Newton's method; then error bounds are computed and utilized in the computation to guarantee that all appropriate triples are found. 
For each triple of values satisfying (1), the program checks whether the element $\eta=c+b \lambda+a \lambda^{2}$ is a unit in $\mathcal{O}_{K}$. When $d=1, \eta$ is obviously in $\mathcal{O}_{K}$ and it suffices to check that the norm of $\eta$ is \pm 1 . When $d>1$, the program also computes the coefficients of $\eta$ in terms of the given basis for $\mathcal{O}_{K}$ and checks that they are rational integers. Of the resulting units, $\bar{\varepsilon}_{i}$ is (by definition) the one with the smallest $i$-th embedding. As described in Section 2, from this process a proper pair of fundamental units for $U_{K}^{+}$is easily obtained. (For consistency in orientation, we choose $\alpha$ and $\beta$ such that the determinant of the $3 \times 3$ matrix determined by $\alpha, 1, \beta$ is positive.) Lemma 1 then yields a fundamental domain, as required.

As explained in Section 3 the subdivision process can be carried out recursively. The key elements of the program are the determination of the subdivision points and the organization and maintenance of the data.

Recall that the subdivision point is an algebraic integer in the set

$$
\left\{t_{1} v_{1}+t_{2} v_{2}+t_{3} v_{3} \mid 0 \leq t_{i}<1\right\}
$$

such that the sum $t_{1}+t_{2}+t_{3}$ is minimal. The point is found using a triply nested loop designed to search through the set in order of increasing sums of coefficients. Each point is tested to see if it is in fact an algebraic integer. The first such algebraic integer found is chosen to be the subdivision point for that cone. (More complicated variations, for example in which a point of minimal trace was chosen from among those of minimal coefficient sum, generally failed to yield simpler resolutions, and so were discarded.)

The data structures used to store information about the cones are carefully constructed to enable easy referencing and updating throughout the program. For each one-dimensional cone, we maintain coordinates in terms of the original root, in terms of the given basis, and in barycentric coordinates for graphing the output. For each two-dimensional cone, we record the vertices of the simplex and the vertices which determine the two three-dimensional cones that border it. For each threedimensional cone, we store the vertices. All of these data must be updated as each new subdivision is made. In each case, vertices that are on the boundary of the original fundamental domain must be tagged, since they frequently need to be treated as special cases.

The program also computes what Cohn refers to as incidence numbers 3 . These complete the combinatorial description of the decomposition. Specifically, let $\gamma_{1}$ and $\gamma_{2}$ be two points determining a two-dimensional cone in the final decomposition of a cusp. The pair of points is then in two three-dimensional cones, say $C\left(\gamma_{1}, \gamma_{2}, \delta_{1}\right)$ and $C\left(\gamma_{1}, \gamma_{2}, \delta_{2}\right)$. Since $\left\{\gamma_{1}, \gamma_{2}, \delta_{1}\right\}$ and $\left\{\gamma_{1}, \gamma_{2}, \delta_{2}\right\}$ must both be bases of $\mathcal{O}_{K}$, there exist positive rational integers $c_{1}$ and $c_{2}$ such that

$$
c_{1} \gamma_{1}+c_{2} \gamma_{2}=\delta_{1}+\delta_{2} .
$$

The numbers $c_{1}$ and $c_{2}$ are the incidence numbers corresponding to the pair $\gamma_{1}, \gamma_{2}$. (For pairs of points contained in one of the original two-dimensional cones, one of the three-dimensional cones is actually an image under the group action of a cone in the decomposed fundamental domain. Although this makes the programming more complicated, the final computation is the same.) 
The computer program is written in $\mathrm{C}++$ as implemented by the GNU $\mathrm{g}++$ compiler and assumes IEEE Floating Point arithmetic. The output used to produce the final graphical output is text designed to be executed by Mathematica, version 2.0 or later. Most integer computations are carried out using 64 bit integer arithmetic; floating point computations use the IEEE double precision data type. The program uses strictly integer computation except during the computation of the units using Theorem 2 (where we specifically corrected for error in the approximations) and in the computing of the graphical output. We encountered no problems with overflow of overly large integers.

The program used in Section 6 to compute values of partial zeta functions was originally written in $\mathrm{C}$, but has been adapted to $\mathrm{C}++$ utilizing a multiprecision data type from the LiDIA C++ library 9 .

The author wishes to thank E. L. Oliver for extensive programming assistance in the early stages of this project.

\section{SAMPle OUtPut}

In this section, we present sample output. In Figures 1 and 2 we present data generated by our program for the two totally real cubic fields of smallest discriminant, and in Figure 3, we present graphical output from the program for two cubic fields with higher discriminants. The input, consisting of a defining cubic polynomial and a basis for the ring of integers, was taken from the table of cubics found in [4.

In Figures 1 and 2, we present first the discriminant, $D$, for the number field and a polynomial any root of which, say $\lambda$, generates the field over $\mathbf{Q}$. We then give the coordinates for the vertices of the simplicial cones: $r, s, t, d$, where the vertex is $\left(r+s \lambda+t \lambda^{2}\right) / d$. In the next table, we give the incidence numbers $c_{1}$ and $c_{2}$ for each two-dimensional cone $C\left(\gamma_{1}, \gamma_{2}\right)$, as described in Section 4, Equation (2). To the right, we present graphical representations of the decompositions. These graphs are generated directly by reading a file generated by the program into Mathematica.

We present two forms of graphical output. The first is a straight-edge version which describes the decomposition as follows. The original fundamental domain is described by a square. Each added vertex is graphed using barycentric coordinates. Pairs of vertices bounding a two-simplex are joined by straight lines. The vertices are numbered in the order that they are found, the first four bounding the original fundamental domain. These numbers correspond to those given in the tables.

The second form is determined by first projecting each point of the fundamental domain along a line through the origin onto the hyperboloid in $\mathbf{R}^{3}$ defined by $x y z=$ 1 , and then taking logarithms. The result is a decomposition of a fundamental domain for the lattice of logarithms of units in the original group of totally positive units. The edges in the graph (which are not straight lines) are the images of the 2-dimensional cones in $\mathbf{R}^{3}$.

In Figure 3, we present the graphical output for two fields of higher discriminant. These illustrate how the resolutions can become more complicated. The decompositions have 20 and 53 vertices, respectively, corresponding to the cubic fields of discriminants 473 and 621 . It should be noted that the number of vertices varies greatly as the discriminant increases. For example, the field of discriminant 564 has 181 vertices in the desingularization, while that of discriminant 697 has only 8 . 


\begin{tabular}{|r|r|r|r|r|}
\hline \multicolumn{5}{|c|}{$D=49$} \\
$x^{3}-7 x^{2}+14 x-7$ \\
\hline$\#$ & $\mathrm{r}$ & $\mathrm{s}$ & $\mathrm{t}$ & $\mathrm{d}$ \\
\hline 1 & -5 & 9 & -2 & 1 \\
2 & 1 & 0 & 0 & 1 \\
3 & 2 & -3 & 1 & 1 \\
4 & -3 & 5 & -1 & 1 \\
5 & 0 & 1 & 0 & 1 \\
6 & -2 & 5 & -1 & 1 \\
\hline
\end{tabular}

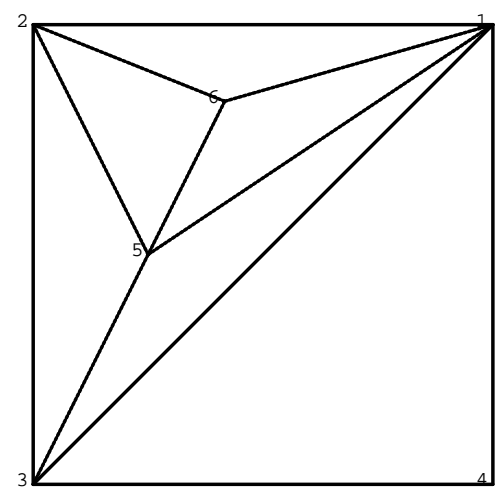

\begin{tabular}{|r|r|r|r|}
\hline$c_{1}$ & $\gamma_{1}$ & $\gamma_{2}$ & $c_{2}$ \\
\hline 0 & 1 & 4 & 4 \\
2 & 2 & 1 & 2 \\
1 & 1 & 3 & 1 \\
2 & 5 & 1 & 0 \\
2 & 5 & 2 & 0 \\
3 & 5 & 3 & -2 \\
2 & 6 & 1 & -1 \\
2 & 6 & 2 & -1 \\
2 & 6 & 5 & -1 \\
\hline
\end{tabular}

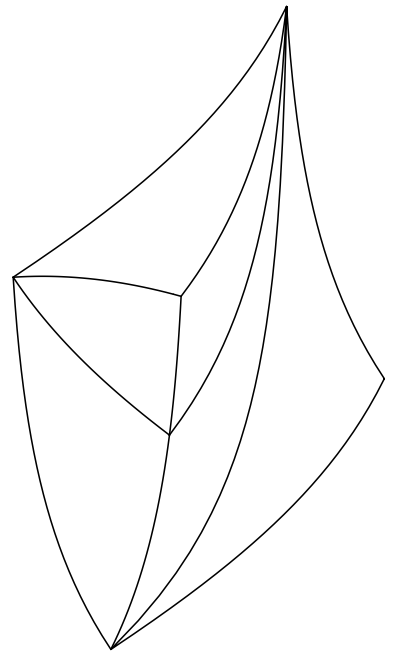

Figure 1. Output for the cubic field of discriminant 49. 


\begin{tabular}{|r|r|r|r|r|}
\hline \multicolumn{5}{|c|}{$D=81$} \\
$x^{3}-6 x^{2}+9 x-1$ \\
\hline$\#$ & $\mathrm{r}$ & $\mathrm{s}$ & $\mathrm{t}$ & $\mathrm{d}$ \\
\hline 1 & 1 & -5 & 2 & 1 \\
2 & 1 & 0 & 0 & 1 \\
3 & 9 & -6 & 1 & 1 \\
4 & 4 & -4 & 1 & 1 \\
5 & 2 & -3 & 1 & 1 \\
6 & 7 & -5 & 1 & 1 \\
7 & 5 & -4 & 1 & 1 \\
8 & 6 & -7 & 2 & 1 \\
\hline
\end{tabular}

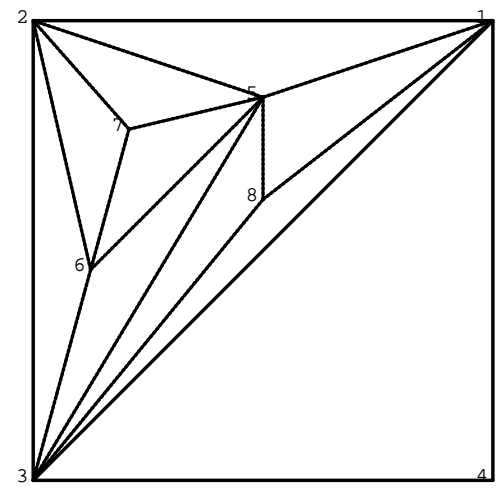

\begin{tabular}{|r|r|r|r|}
\hline$c_{1}$ & $\gamma_{1}$ & $\gamma_{2}$ & $c_{2}$ \\
\hline 1 & 1 & 4 & 3 \\
0 & 2 & 1 & 4 \\
1 & 1 & 3 & 1 \\
4 & 5 & 1 & -1 \\
3 & 5 & 2 & 0 \\
2 & 5 & 3 & 1 \\
2 & 6 & 2 & 0 \\
3 & 6 & 3 & -2 \\
2 & 6 & 5 & 0 \\
2 & 7 & 5 & -1 \\
2 & 7 & 2 & -1 \\
2 & 7 & 6 & -1 \\
2 & 8 & 3 & -1 \\
2 & 8 & 1 & -1 \\
2 & 8 & 5 & -1 \\
\hline
\end{tabular}

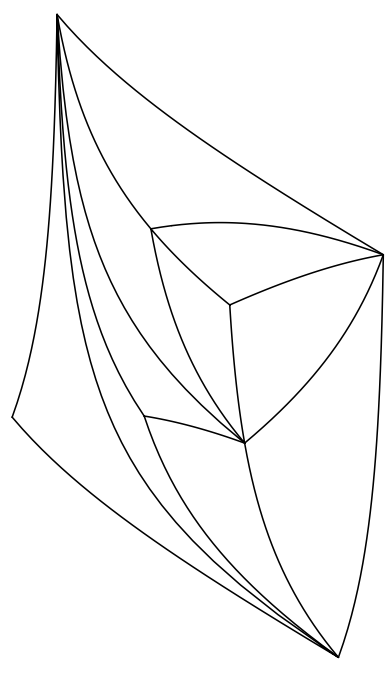

Figure 2. Output for the cubic field of discriminant 81 . 

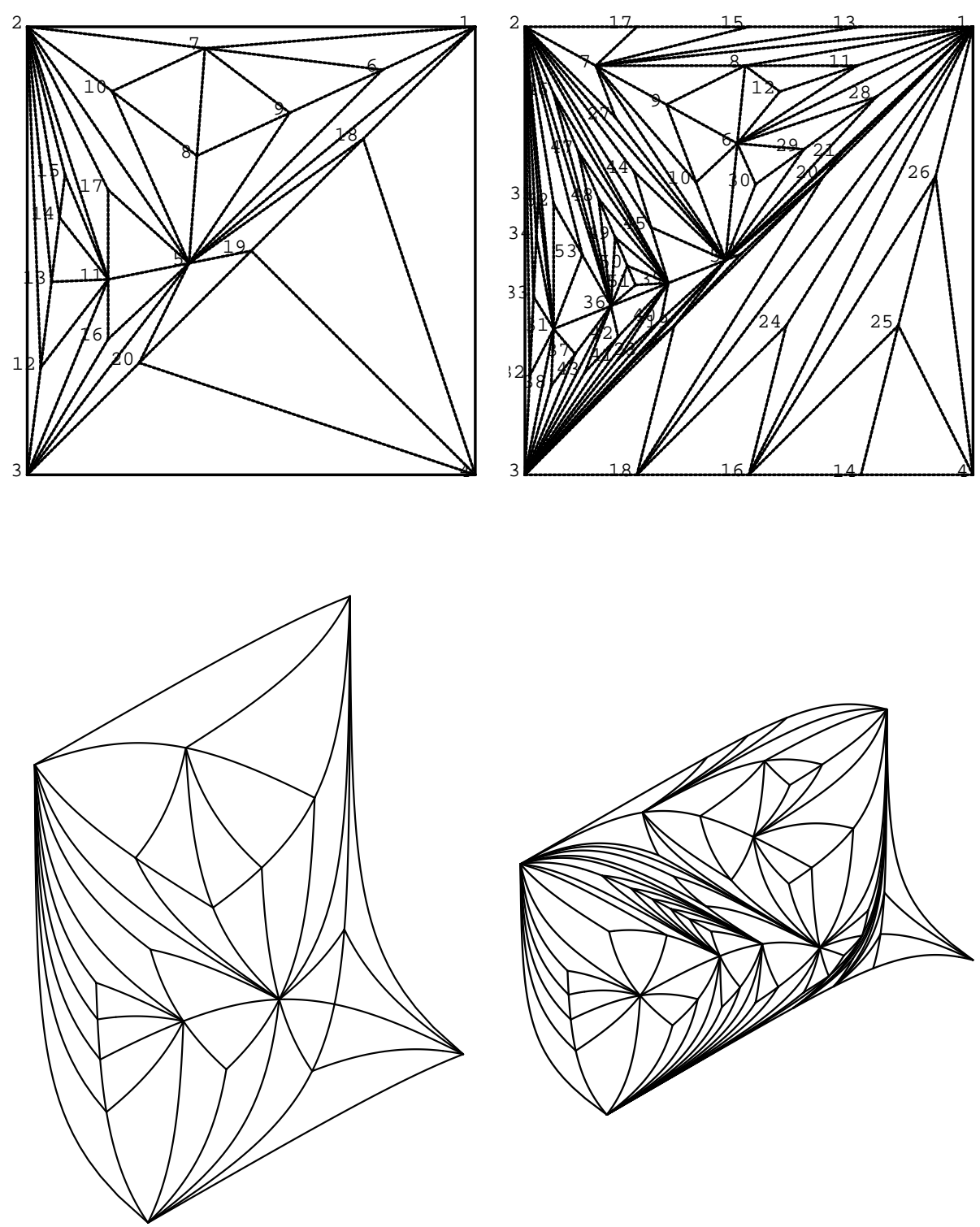

Figure 3. Graphical output for fields of discriminants 473 and 621

\section{Zeta FunCtions}

In this section, we give an application of the above program to the problem of computing values of partial zeta functions of totally real cubic fields evaluated at -1 . We start by defining the zeta functions and explaining Shintani's method [12] for computing special values. Since we are only dealing with cubic number fields, we simplify to that case throughout. 
Let $\mathfrak{f}$ and $\mathfrak{b}$ be relatively prime ideals in $\mathcal{O}_{K}$. For $\Re(s)>1$ the partial zeta function $\zeta(\mathfrak{b}, \mathfrak{f}, s)$ is defined by

$$
\zeta(\mathfrak{b}, \mathfrak{f}, s)=\sum_{\mathfrak{g}} N(\mathfrak{g})^{-s}
$$

where the summation is taken over all ideals $\mathfrak{g}$ in the same narrow ray class modulo $\mathfrak{f}$ as $\mathfrak{b}$. For all other $s \neq 1$, the function is defined by analytic continuation.

Let $U(\mathfrak{f})_{K}^{+}$denote the totally positive units of $\mathcal{O}_{K}$ that are congruent to 1 modulo $\mathfrak{f}$. Fix a finite system $\left\{C_{j}\right\}_{j \in J}$, of open simplicial cones, forming a fundamental domain for the action of $U(\mathfrak{f})_{K}^{+}$on $\mathbf{R}_{+}^{3}$. For each cone $C_{j}=C_{j}\left(v_{j 1}, v_{j 2}, \ldots, v_{j r(j)}\right)$ and for independent variables $t_{1}, t_{2}, t_{3}$, let

$$
L_{\ell}(t)=v_{j \ell}^{(1)} t_{1}+v_{j \ell}^{(2)} t_{2}+v_{j \ell}^{(3)} t_{3}, \quad 1 \leq \ell \leq r(j) .
$$

Let $x=\left(x_{1}, \ldots, x_{r(j)}\right)$ be an $r(j)$-tuple of real numbers and let $u$ be an independent variable. Following Shintani, we let $(m !)^{-3} B_{m}\left(C_{j}, x\right)^{(k)}$ be the coefficient of $u^{3(m-1)}\left(t_{1} \cdots t_{k-1} t_{k+1} \cdots t_{3}\right)^{m-1}$ in the Laurent expansion at the origin of the function

$$
F(u, t)=\left.\prod_{\ell=1}^{r} \frac{e^{u x_{\ell} L_{\ell}(t)}}{e^{u L_{\ell}(t)}-1}\right|_{t_{k}=1}
$$

Further, let

$$
B_{m}\left(C_{j}, x\right)=\frac{1}{3} \sum_{k=1}^{3} B_{m}\left(C_{j}, x\right)^{(k)} .
$$

For $S \subseteq K$, let $R(j, S)$ denote the set of all $r(j)$-tuples of rational numbers $x=\left(x_{1}, \ldots, x_{r(j)}\right)$ such that $0<x_{i} \leq 1$, for each $i$, and

$$
x_{1} v_{j 1}+x_{2} v_{j 2}+\cdots+x_{r(j)} v_{j r(j)} \in S .
$$

Finally, let $\mathfrak{b}^{-1} \mathfrak{f}+1$ denote the set of all numbers $x \in K$ such that $x-1 \in \mathfrak{b}^{-1} \mathfrak{f}$. The following theorem is proved in [12].

Theorem 3 (Shintani). Using the above notation,

$$
\zeta_{k}(\mathfrak{b}, \mathfrak{f}, 1-m)=m^{-3} N(\mathfrak{b})^{m-1} \sum_{j \in J} \sum_{x \in R\left(j, \mathfrak{b}^{-1} \mathfrak{f}+1\right)}(-1)^{r(j)} B_{m}\left(C_{j}, x\right) .
$$

Simplifying to the case $\mathfrak{f}=\mathcal{O}_{K}$ and $m=2$ yields

$$
\zeta\left(\mathfrak{b}, \mathcal{O}_{K},-1\right)=\frac{1}{8} N(\mathfrak{b}) \sum_{j \in J} \sum_{x \in R\left(j, \mathfrak{b}^{-1}\right)}(-1)^{r(j)} B_{2}\left(C_{j}, x\right)
$$

The author developed software [6] to compute the values of $\zeta\left(\mathfrak{b}, \mathcal{O}_{K},-1\right)$ for totally real cubic fields, based on Equation (3). The program described here provides specifications of the simplicial cones needed as input to the program. To compute $\zeta\left(\mathcal{O}_{K}, \mathcal{O}_{K},-1\right)$, no input is required beyond the coefficients of a polynomial and an integral basis given in terms of a root of that polynomial. Although the decomposition is not necessary for the use of Shintani's method, the computation is greatly simplified by its use. Specifically, once the domain is decomposed as described earlier in this paper, the innermost summation of Equation (3) reduces to a single summand because there is only one valid point to be considered. With minor 
TABLE 1. Values of $\zeta\left(\mathcal{O}_{K}, \mathcal{O}_{K},-1\right)$

\begin{tabular}{||r|c||c|c||r|c||r|r||r|c||}
\hline $\mathrm{D}$ & $\zeta(-1)$ & $\mathrm{D}$ & $\zeta(-1)$ & $\mathrm{D}$ & $\zeta(-1)$ & $\mathrm{D}$ & $\zeta(-1)$ & $\mathrm{D}$ & $\zeta(-1)$ \\
\hline 49 & $-1 / 21$ & 469 & -2 & 788 & $-7 / 3$ & 1129 & $-11 / 3$ & 1425 & $-29 / 3$ \\
81 & $-1 / 9$ & 473 & $-5 / 3$ & 837 & $-16 / 3$ & 1229 & $-14 / 3$ & 1436 & $-22 / 3$ \\
148 & $-1 / 3$ & 564 & -3 & 892 & $-10 / 3$ & 1257 & -4 & 1489 & -4 \\
169 & $-1 / 3$ & 568 & $-10 / 3$ & 940 & $-22 / 3$ & 1300 & -9 & 1492 & $-17 / 3$ \\
229 & $-1 / 3$ & 621 & $-10 / 3$ & 961 & $-28 / 3$ & 1304 & $-38 / 3$ & 1509 & -7 \\
257 & $-1 / 3$ & 697 & $-4 / 3$ & 985 & $-7 / 3$ & 1345 & $-23 / 3$ & 1524 & $-41 / 3$ \\
316 & $-4 / 3$ & 733 & -4 & 993 & $-17 / 3$ & 1369 & -7 & 1556 & $-19 / 3$ \\
321 & -1 & 756 & $-13 / 3$ & 1016 & $-13 / 3$ & 1373 & $-34 / 3$ & 1573 & $-38 / 3$ \\
361 & -1 & 761 & $-5 / 3$ & 1076 & $-11 / 3$ & 1384 & $-19 / 3$ & 1593 & $-16 / 3$ \\
404 & $-5 / 3$ & 785 & $-11 / 3$ & 1101 & $-26 / 3$ & 1396 & $-16 / 3$ & 1620 & $-43 / 3$ \\
\hline
\end{tabular}

modifications to the older software (in order to increase the precision) we computed the values of $\zeta\left(\mathcal{O}_{K}, \mathcal{O}_{K},-1\right)$ for the fifty totally real cubic fields of smallest discriminant. The results are displayed in Table 1 .

\section{REFERENCES}

[1] A. Ash, D. Mumford, D. Rapoport, and Y. Tai. Smooth compactifications of locally symmetric varieties. Mathematical Sciences Press, Brookline, MA, 1975. MR 56:15642

[2] W. E. H. Berwick. Algebraic number fields with two independent units. Proc. London Math. Soc., 34:360-378, 1932

[3] H. Cohn. Formal ring of a cubic solid angle. J. Number Theory, 10:135-150, 1978. MR 58:16513

[4] T. W. Cusick and L. Schoenfeld. A table of fundamental pairs of units in totally real cubic fields. Math. Comput., 48(177):147-158, 1987. MR 87k:11123

[5] F. Ehlers. Eine Klasse komplexer Mannigfaltigkeiten und die Auflösung einiger isolierter Singularitäten. Math. Ann., 218:127-156, 1975. MR 58:11502

[6] H. G. Grundman. The arithmetic genus of Hilbert modular varieties over non-Galois cubic fields. J. Number Theory, 37:343-365, 1991. MR 92d:11047

[7] C. Haspel and A. Vasquez. Toroidal structures associated with totally real cubic number fields. Technical Report TR 73-026, IBM Systems Research Institute, New York, 1983.

[8] F. Hirzebruch. The Hilbert modular group, resolutions of the singularities at the cusps and related problems. In Friedrich Hirzebruch: Gesammelte Abhandlungen, Collected Papers, Band II, pages 192-205. Springer-Verlag, Berlin, 1987. MR 89h:01106

[9] LiDIA-Group. LiDIA - A library for computational number theory. Universität des Saarlandes, 1995.

[10] M. Pohst and H. Zassenhaus. Algorithmic Algebraic Number Theory. Cambridge University Press, Cambridge, 1989. MR 92b:11074

[11] I. Satake. On the arithmetic of tube domains. Bull. Am. Math. Soc., 79:1076-1094, 1973. MR 48:8861

[12] T. Shintani. On evaluation of zeta functions of totally real algebraic number fields at nonpositive integers. J. Fac. Sci., Univ. Tokyo, Sect. I A, 23(2):393-417, 1976. MR 55:266

[13] E. Thomas. Fundamental units for orders in certain cubic number fields. J. Reine Angew. Math., 310:33-55, 1979. MR 81b:12009

[14] E. Thomas and A. T. Vasquez. On the resolution of cusp singularities and the Shintani decomposition in totally real cubic number fields. Math. Ann., 247:1-20, 1980. MR 81h:10037

Department of Mathematics, Bryn Mawr College, Bryn Mawr, PA 19010

E-mail address: grundman@brynmawr.edu 